\title{
Heated Permeable Stretching Surface in a Porous Medium Using Nanofluids
}

\author{
M. Sheikholeslami ${ }^{\dagger}$ and D. D. Ganji \\ Department of Mechanical Engineering, Babol University of Technology, Babol, P.O. Box 484, Islamic \\ Republic of Iran. \\ †Corresponding Author Email: Mohsen.sheikholeslami@yahoo.com,
}

(Received August 5, 2013; accepted August 28, 2013)

\begin{abstract}
In this article, two-dimensional laminar-forced convection nanofluids flow over a stretching surface in a porous medium has been studied. The governing partial differential equations with the corresponding boundary conditions are reduced to a set of ordinary differential equations with the appropriate boundary conditions using similarity transformation, which is then solved numerically by the fourth order Runge-Kutta integration scheme featuring a shooting technique. Different models of nanofluid based on different formulas for thermal conductivity and dynamic viscosity are used. Different types of nanoparticles as copper, silver, alumina and titanium Oxide with water and Ethylene glycol as their base fluids has been considered. The influence of significant parameters such as nanoparticle volume fraction, kind of nanofluid, Magnetic parameter and Reynolds number on the flow and heat transfer characteristics is discussed. The influence of significant parameters such as Thermal conductivity parameter, volume fraction of the nanoparticles, Permeability parameter, suction/injection parameter and Velocity ratio parameter on the flow and heat transfer characteristics is discussed. It was found that choosing Titanium oxide as the nanoparticle and Ethylene glycol as base fluid proved to have the highest cooling performance for this problem.
\end{abstract}

Keywords: Nanofluid, Variable thermal conductivity, Porous media, Stagnation point flow, Stretching sheet, Force convection.

NOMENCLATURE

$\begin{array}{llll}A_{l}, A_{2}, A_{3} & \text { constants parameters } & \eta & \text { similarity parameter } \\ a & \text { stretching sheet parameter } & \theta & \text { similarity function for temperature } \\ b & \text { free stream velocity parameter } & \rho & \text { fluid density } \\ C_{f} & \text { skin friction coefficient } & \phi & \text { nanoparticle volume fraction } \\ f & \text { dimensionless stream function } & \mu & \text { dynamic viscosity } \\ k & \text { thermal conductivity } & v & \text { kinematic viscosity } \\ k^{*} & \text { variable thermal conductivity } & \varepsilon & \text { thermal conductivity parameter } \\ K & \text { permeability of the porous medium } & \tau_{w} & \text { wall shear stress } \\ K_{l} & \text { permeability parameter } & \psi & \text { stream function } \\ N u & \text { Nusselt number } & \lambda & \text { velocity ratio parameter } \\ P r & \text { Prandtl number } & S u b s c r i p t s & \\ q_{w} & \text { surface heat flux } & w & \text { condition at the surface } \\ \mathrm{Re}_{x} & \text { local Reynolds number } & \infty & \text { condition at infinity } \\ T & \text { fluid temperature } & n f & \text { nanofluid } \\ \text { Greek symbols } & & f & \text { base fluid } \\ \alpha & \text { thermal diffusivity } & s & \text { nano-solid-particles }\end{array}$




\section{INTRODUCTION}

Fluid heating and cooling are significant in many industries and engineering applications such as power generating, manufacturing and transportation and in particular in thin film solar energy collector devices. Convectional heat transfer fluids, including oil, water, and ethylene glycol mixture are poor heat transfer fluids, since the thermal conductivity of these fluids play important role on the heat transfer coefficient between the heat transfer medium and the heat transfer surface. In comparison, metals have thermal conductivities up to three times higher than these fluids, so it is naturally desirable to combine the two substances to produce a heat transfer medium that behaves like a fluid, but has the thermal conductivity of a metal. The term "nanofluid" refers to a liquid containing a suspension of submicronic solid particles (nanoparticles). The term was coined by Choi (1995). Sheikholeslami et al. (2012, a) studied the natural convection in a concentric annulus between a cold outer square and heated inner circular cylinders in presence of static radial magnetic field. They reported that average Nusselt number is an increasing function of nanoparticle volume fraction as well as Rayleigh number, while it is a decreasing function of Hartmann number. Ramiar et al. (2012) studied the effect of Axial Conduction and Variable Properties on Two-Dimensional conjugate Heat Transfer. They found that considering variable properties will cause severe changes to the Nusselt number distribution especially for low Reynolds numbers. Sheikholeslami et al. (2012, b) investigated natural convection of nanofluids in an enclosure between a circular and a sinusoidal cylinder in the presence of magnetic field. Soleimani et al. (2012) studied natural convection heat transfer in a semi-annulus enclosure filled with nanofluid. They found that the angle of turn has an important effect on the streamlines, isotherms and maximum or minimum values of local Nusselt number. Sheikholeslami et al. (2013, a) used the lattice Boltzmann method to examine free convection of nanofluids. They found that choosing copper as the nanoparticle leads to obtain the highest enhancement for this problem. The problem of steady, laminar, mixed convection flow of a nonNewtonian fluid past a preamble vertical flat plate embedded in a porous medium saturated with a nanofluid was considered by Rashad et al. (2013). They showed that the effects of Lewis number, Brownian motion or thermophoresis parameters are more pronounced on the local Sherwood number than on the local Nusselt number. Sheikholeslami et al. (2013, b) performed a numerical study to investigate natural convection in a square cavity with curve boundaries filled with $\mathrm{Cu}$-water nanofluid. Their results proved that the change of inclination angle has a significant impact on the thermal and hydrodynamic flow fields. Recently several authors investigated about natural convection and effect of adding nanoparticle in the base fluid on flow and heat transfer (Khan et al. (2011), Vajravelu et al. (2011), Sheikholeslami et al. (2013, c), Sheikholeslami et al. (2013, d),
Sheikholeslami et al. (2013, e), Yacob et al. (2011), Ellahi (2013)).

The study of convective heat transfer in fluidsaturated porous media has many important applications in technology geothermal energy recovery such as oil recovery, food processing, fiber and granular insulation, porous burner and heater, combustion of low-calorific fuels to diesel engines and design of packed bed reactors. In general, suction tends to increase the skin friction and heat transfer coefficients, whereas injection acts in the opposite manner (Al-Sanea (2004)). Study of heat transfer in boundary layer over stretching surface finds applications in extrusion of plastic sheets, polymer, spinning of fibers, cooling of elastic sheets, etc. The quality of final product depends on the rate of heat transfer and therefore cooling procedure has to be controlled effectively. Afzal and Varshney (1980) proposed a general power-law stretching sheet of the form $x^{m}$, where $x$ is the distance measured from the issuing slit and $m$ is a constant. The heat transfer from a stretching sheet with variable surface temperature was studied by Afzal (1993), who found exact solutions for specific values of $m$. Fluid flow and heat transfer characteristics on stretching sheet with variable temperature condition have been investigated by Grubka and Bobba (1985) with variable temperature. Heat transfer of a nanofluid flow which is squeezed between parallel plates was investigated analytically by Sheikholeslami and Ganji (2013). Ghasemi et al. (2012) studied the natural convection heat transfer in a cold outer circular enclosure containing a hot inner elliptic cylinder. They found that an inclination angle of cylinder is an important factor in heat transfer enhancement. The entropy generation process in the bypass transition scenario was investigated for a flat plate boundary layer by Ghasemi et al. (2013). CVFEM has been applied to investigate flow and heat transfer of $\mathrm{CuO}$-water nanofluid in presence of magnetic field by Sheikholeslami et al. (2013,f). Sheikholeslami et al. $(2013$, g) studied the effect of a magnetic field on natural convection in an inclined half-annulus enclosure filled with $\mathrm{Cu}-$ water nanofluid. Their results indicated that Hartmann number and the inclination angle of the enclosure can be considered as control parameters at different Rayleigh number.

In the present paper two-dimensional laminarforced convection flow of a nanofluid over a stretching porous surface has been studied. The reduced ordinary differential equations are solved numerically using the fourth order Runge-Kutta. According to wide application of stretching surface, effect of velocity ratio parameter is investigated. The type of nanofluid is a key factor for heat transfer enhancement. So different types of nanoparticles and base fluids (Copper, Silver, Alumina and Titanium Oxide with water and Ethylene glycol) are used to find the highest values of percentage of heat transfer enhancement. Models of nanofluid based on different formulas for thermal conductivity and dynamic viscosity (cylindrical shaped nanoparticles) is considered in this study to 
choose the best model. Also effects of variable thermal conductivity, permeability parameter, suction/injection parameter on flow and heat transfer are examined.

\section{PROBLEM FORMULATION}

Consider the steady, two-dimensional flow of a nanofluid near the stagnation point on a stretching sheet saturated at a porous surface (highly permeable) as shown in Fig. 1.

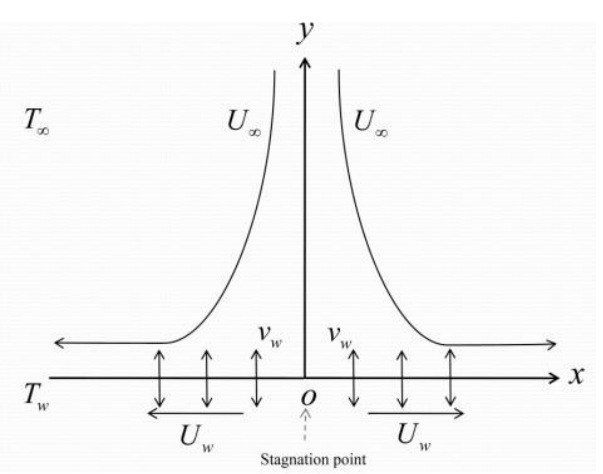

Fig. 1. Figure of geometery.

The stretching velocity $U_{w}(x)$ and the free stream velocity $U_{\infty}(x)$ are assumed to vary proportional to the distance $x$ from the stagnation point, i.e. $U_{w}(x)=a x$ and $U_{\infty}(x)=b x, \quad$ where $a$ and $\quad b$ are constants with $a>0$ and $b \geq 0$. It is assumed that the temperature at the stretching surface takes the constant values $T_{w}$, while the temperature of the ambient nanofluid, attained as $y$ tends to infinity, takes the constant values $T_{\infty}$. The fluid is a water based nanofluid containing different types of nanoparticles: $\mathrm{Cu}, \mathrm{Al}_{2} \mathrm{O}_{3}, \mathrm{Ag}$ and $\mathrm{TiO}_{2}$. It is assumed that the base fluid and the nanoparticles are in thermal equilibrium and no slip occurs between them. The thermo physical properties of the nanofluid are given in Table 1 .

Table 1 Thermo physical properties of water and nanoparticles

\begin{tabular}{|c|c|c|c|}
\hline & $\begin{array}{c}\rho \\
\left(\mathrm{kg} / \mathrm{m}^{\wedge} 3\right)\end{array}$ & $C_{p}(j / k g k)$ & $\begin{array}{c}\mathrm{k} \\
(\mathrm{w} / \mathrm{mk})\end{array}$ \\
\hline water & 997.1 & 4179 & 0.613 \\
\hline $\begin{array}{c}\text { Ethylene } \\
\text { glycol }\end{array}$ & 1115.6 & 2386.5 & 0.24998 \\
\hline $\mathrm{Cu}$ & 8933 & 385 & 401 \\
\hline $\mathrm{Ag}$ & 10500 & 235 & 429 \\
\hline $\mathrm{Al}_{2} \mathrm{O}_{3}$ & 3970 & 765 & 40 \\
\hline $\mathrm{TiO}_{2}$ & 4250 & 686.2 & 8.9538 \\
\hline
\end{tabular}

Under these assumptions:

$\frac{\partial u}{\partial x}+\frac{\partial v}{\partial y}=0$,

$\rho_{n f}\left(u \frac{\partial u}{\partial x}+v \frac{\partial u}{\partial y}-U_{\infty} \frac{d U_{\infty}}{d x}\right)=$

$\mu_{n f} \frac{\partial^{2} u}{\partial y^{2}}+\frac{\mu_{n f}}{K}\left(U_{\infty}-u\right)$, $\left(\rho C_{p}\right)_{n f}\left(u \frac{\partial T}{\partial x}+v \frac{\partial T}{\partial y}\right)=\frac{\partial}{\partial y}\left(k_{n f}^{*} \frac{\partial T}{\partial y}\right)$,

$k_{n f}^{*}=k_{n f}(1+\varepsilon \theta)$

Subject to the boundary conditions

$y=0: \quad u=U_{w}(x), \quad v=v_{w}, \quad T=T_{w}$

$y \rightarrow \infty: u \rightarrow U_{\infty}(x), T \rightarrow T_{\infty}$

where $u$ and $v$ are the velocity components along the $x$ and $y$ axes, respectively, $T$ is fluid temperature, $\varepsilon$ is Thermal conductivity parameter and $K$ is the permeability of the porous medium. Also, $v_{w}$ is the wall mass flux with $v_{w}<0$ for suctions and $v_{w}>0$ for injection, respectively. The effective density $\rho_{n f}$, the effective dynamic viscosity $\mu_{n f}$, the heat capacitance $\left(\rho C_{p}\right)_{n f}$ and they are given as:

$\rho_{n f}=\rho_{f}(1-\phi)+\rho_{s} \phi$

$\mu_{n f}=\frac{\mu_{f}}{(1-\phi)^{2.5}}$

$\left(\rho C_{p}\right)_{n f}=\left(\rho C_{p}\right)_{f}(1-\phi)+\left(\rho C_{p}\right)_{s} \phi$

Effective thermal conductivity $\left(k_{n f}\right)$ can be incorporated from the following expression:

$\frac{k_{n f}}{k_{f}}=\frac{k_{s}+(n-1) k_{f}-(n-1) \phi\left(k_{f}-k_{s}\right)}{k_{s}+(n-1) k_{f}+\phi\left(k_{f}-k_{s}\right)}$

where $n$ is the empirical shape factor for the nanoparticle. In particular, $n=3$ for spherical shaped nanoparticles and $n=3 / 2$ for cylindrical ones. Here, $\phi$ is the solid volume fraction, $\mu_{f}$ is the dynamic viscosity of the base fluid, $\rho_{f}$ and $\rho_{s}$ are the densities of the base fluid and nanoparticle, respectively, $k_{f}$ and $k_{s}$ are the thermal conductivities of the base fluid and nanoparticle, respectively. Models of nanofluid based on different formulas for thermal conductivity and dynamic viscosity is shown in Table 2.

Table 2 Models of nanofluid based on different formulas for thermal conductivity and dynamic viscosity.

\begin{tabular}{|c|c|}
\hline Model $I$ & Model $I I$ \\
\hline \multicolumn{2}{|c|}{ Shape of nanoparticles } \\
\hline Spherical & Cylindrical (nanotubes) \\
\hline \multicolumn{2}{|c|}{ Thermal conductivity } \\
\hline$\frac{k_{n f}}{k_{f}}=\frac{k_{s}+2 k_{f}-2 \phi\left(k_{f}-k_{s}\right)}{k_{s}+2 k_{f}+\phi\left(k_{f}-k_{s}\right)}$ & $\frac{k_{n f}}{k_{f}}=\frac{k_{s}+(1 / 2) k_{f}-(1 / 2) \phi\left(k_{f}-k_{s}\right)}{k_{s}+(1 / 2) k_{f}+\phi\left(k_{f}-k_{s}\right)}$ \\
\hline \multicolumn{2}{|c|}{ Dynamic viscosity } \\
\hline \multicolumn{2}{|c|}{$\mu_{n f}=\frac{\mu_{f}}{(1-\phi)^{25}}$} \\
\hline
\end{tabular}

The continuity Eq (1) is satisfied by introducing a stream function $\psi$ such that

$u=\frac{\partial \psi}{\partial y} \quad$ and $\quad v=-\frac{\partial \psi}{\partial x}$.

The momentum and energy equations can be transformed into the corresponding ordinary differential equations by the following transformation: 
M. Sheikholeslami and D. D. Ganji / JAFM, Vol. 7, No. 3, pp. 535-542, 2014.

$\eta=\left(\frac{a}{v}\right)^{1 / 2} y, f(\eta)=\frac{\psi}{(a v)^{1 / 2} x}, \quad \theta(\eta)=\frac{T-T_{\infty}}{T_{w}-T_{\infty}}$

Using model $I$, the transformed ordinary differential equations are:

$$
\begin{aligned}
& f^{\prime \prime \prime}+f f^{\prime \prime}-f^{\prime 2}+\lambda^{2}+\frac{K_{I}}{A_{I} \cdot(1-\phi)^{2.5}}\left(\lambda-f^{\prime}\right)=0, \\
& \frac{1}{\operatorname{Pr}} \cdot \frac{A_{1} \cdot A_{3} \cdot(1-\phi)^{2.5}}{A_{2}}\left((1+\varepsilon \theta) \theta^{\prime \prime}+\varepsilon\left(\theta^{\prime}\right)^{2}\right)+f \theta^{\prime}=0 .
\end{aligned}
$$

subject to the boundary conditions (4) which become

$$
\begin{array}{ll}
f(0)=\frac{-v_{w}}{(a v)^{0.5}}=\gamma, & f^{\prime}(0)=1, \quad \theta(0)=1, \\
f^{\prime}(\infty) \rightarrow \lambda, & \theta(\infty) \rightarrow 0 .
\end{array}
$$

Here prime denote differentiation with respect to $\eta$ ,$\lambda=b / a$ is the Velocity ratio parameter, $\operatorname{Pr}=\left(\mu_{f}\left(\rho C_{p}\right)_{f}\right) /\left(\rho_{f} k_{f}\right)$ is the Prandtl number and $K_{l}=\mu_{f} /\left(\rho_{f} K a\right)$ is the Permeability parameter and $A_{1}, A_{2}, A_{3}$ are parameters having the following form:

$A_{l}=(1-\phi)+\frac{\rho_{s}}{\rho_{f}} \phi$

$A_{2}=(1-\phi)+\frac{\left(\rho C_{p}\right)_{s}}{\left(\rho C_{p}\right)_{f}} \phi$

$A_{3}=\frac{k_{n f}}{k_{f}}=\frac{k_{s}+2 k_{f}-2 \phi\left(k_{f}-k_{s}\right)}{k_{s}+2 k_{f}+\phi\left(k_{f}-k_{s}\right)}$

The quantities of practical interest in this study are the skin friction coefficient $\left(C_{f}\right)$ and the local Nusselt number $\left(N u_{x}\right)$, which are defined as

$$
C_{f}=\frac{\mu_{n f}}{\rho_{f} U_{w}^{2}}\left(\frac{\partial u}{\partial y}\right)_{y=0}, N u=\frac{x k_{n f}}{k_{f}\left(T_{w}-T_{\infty}\right)}\left(-\frac{\partial T}{\partial y}\right)_{y=0},
$$

with $k_{n f}$ being the thermal conductivity of the nanofluid. Therefore, the skin friction coefficient and the local Nusselt number can be expressed as $\tilde{C}_{f}=\frac{1}{2} C_{f} \operatorname{Re}_{x}^{0.5}=\frac{1}{(1-\phi)^{2.5}} f^{\prime \prime}(0)$,

$\tilde{N} u_{x}=N u_{x} \operatorname{Re}_{x}^{-0.5}=-\frac{k_{n f}}{k_{f}} \theta^{\prime}(0)$,

where $R e_{x}=\rho_{f} U_{w} x / \mu_{f}$ is the local Reynolds number based on the stretching velocity. The quantities $\tilde{C}_{f}$ and $\tilde{N} u_{x}$ are referred as the reduced skin friction coefficient and reduced local Nusselt number, respectively.

\section{RESULTS AND DISCUSSION}

The ordinary differential equations with the boundary conditions have been solved numerically for some values of the governing parameters namely: thermal conductivity parameter, volume fraction of the nanoparticles, permeability parameter, suction/injection parameter, velocity ratio parameter and different kinds of nanoparticles using the fourth order Runge-Kutta. It is seen from Table 3 that the numerical values of $-\theta^{\prime}(0)$ in the present paper are in agreement with those obtained by Sharma and Singh (2009).

Table 3 Values of $-\theta^{\prime}(0)$ for different values of $\lambda$ are compared with the results obtained by Sharma and Singh (2009)

when $M=0, S=0, \varepsilon=0, \operatorname{Pr}=0.05, \phi=0$, and $K_{l}=0$.

\begin{tabular}{|c|c|c|}
\hline$\lambda$ & \multicolumn{2}{|c|}{$-\theta^{\prime}(0)$} \\
\cline { 2 - 3 } & $\begin{array}{c}\text { Sharma and } \\
\text { Singh } \\
(2009)\end{array}$ & $\begin{array}{c}\text { Present } \\
\text { work }\end{array}$ \\
\hline 0.1 & 0.81245 & 0.0811 \\
\hline 0.5 & 0.135571 & 0.1354 \\
\hline 2 & 0.241025 & 0.2412 \\
\hline
\end{tabular}

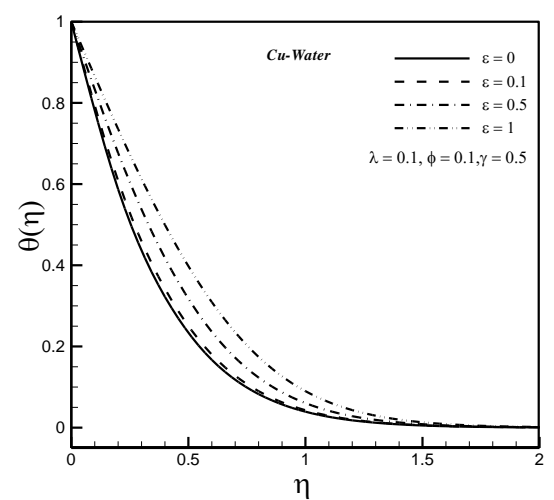

(a)

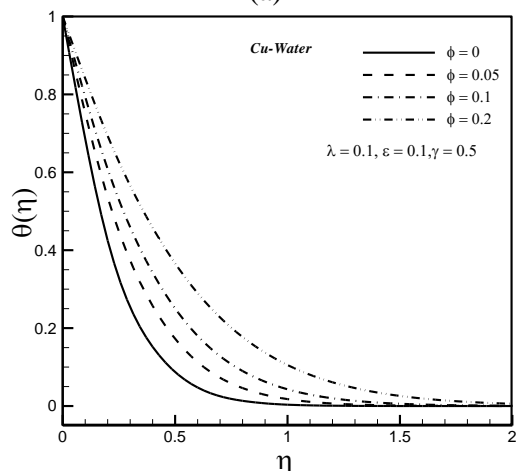

(b)

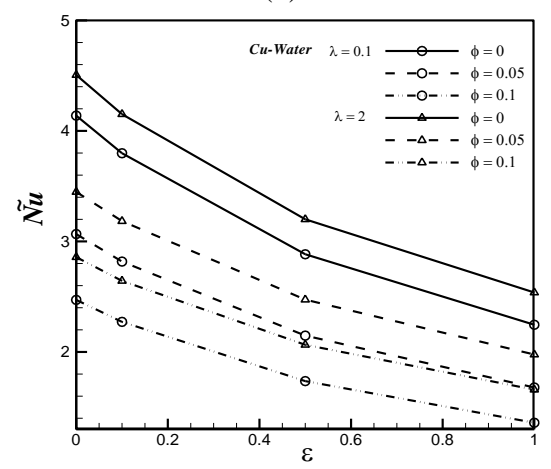

(c)

Fig. 2. Effect of Thermal conductivity parameter $(\varepsilon)$ and nanoparticle volume fraction $(\phi)$ on

Temperature distribution and Nusselt number when $K_{I}=0.1$ and $\operatorname{Pr}=6.2$. 
Effects of Thermal conductivity parameter and nanoparticle volume fraction on Temperature distribution and Nusselt number is shown in Fig.2. Because of increasing in the thermal conductivity due to increase in $\varepsilon$, the thermal boundary layer thickness increases, thus decreases heat transfer rate at the surface. When the volume fraction of the nanoparticles increases from 0 to 0.2 , the thermal boundary layer is increased. This agrees with the physical behavior in that when the volume fraction of copper increases the thermal conductivity increases, and then the thermal boundary layer thickness increases, hence increases heat transfer rate at the surface. Figure 3 is graphical representation of the effect of the suction/injection parameter for $\mathrm{Cu}$-water on velocity profile and temperature distribution. We know that the effect of suction is to bring the fluid closer to the surface and, therefore, to reduce the thermal boundary layer thickness and in turn increases the Nusselt number, but opposite trend is observed for injection. When stronger injection is provided, the heated $\mathrm{Cu}$-water is pushed less from the wall than for a regular fluid $(\phi=0)$, i.e. the existence of the nanoparticle leads to a small increase of the velocity profiles, but for suction, it is noted an opposite behavior. It is clear that the thermal boundary layer thickness for the injection case is greater than for suction. When $\lambda>1$, the flow has a boundary layer structure and when $\lambda<1$ the flow has an inverted boundary layer structure, which results from the fact that when $(b / a)<1$, the stretching velocity $a x$ of the surface exceeds the velocity $b x$ of the external stream. It is to be noted that no momentum boundary layer is formed when $\lambda=1$. It is seen from Fig. 3, the thermal boundary layer thickness for $\lambda<1$ is greater than that of $\lambda>1$. For $\lambda=0.1$, the effect of suction is to decrease the velocity profile, whereas the effect of injection is to increase this profile. Also it can be found that for both cases, all boundary layer thicknesses increase with increasing values of suction/injection parameter.

Table 4 Effects of Permeability parameter, Suction/injection parameter, Velocity ratio parameter and nanoparticle volume fraction on skin friction coefficient when $\varepsilon=0.1$ and $P r=6.2$ for $\mathrm{Cu}$-Water.

\begin{tabular}{|c|c|c|c|c|c|}
\hline \multirow{2}{*}{$K_{l}$} & \multirow{2}{*}{$\gamma$} & \multicolumn{2}{|c|}{$\lambda=0.1$} & \multicolumn{2}{c|}{$\lambda=2$} \\
\cline { 3 - 6 } & & \multicolumn{4}{|c|}{$\phi$} \\
\cline { 3 - 6 } & & 0 & 0.05 & 0 & 0.05 \\
\hline 0.1 & -0.5 & -0.80524 & -0.79792 & 1.778452 & 1.773849 \\
\hline 0.1 & 0.5 & -1.26387 & -1.25665 & 2.333717 & 2.329335 \\
\hline 1 & 0.5 & -1.5677 & -1.5104 & 2.535495 & 2.495021 \\
\hline 0.5 & -0.5 & -0.95151 & -0.91898 & 1.874645 & 1.852573 \\
\hline 1 & -0.5 & -1.11245 & -1.05467 & 1.989145 & 1.947061 \\
\hline
\end{tabular}

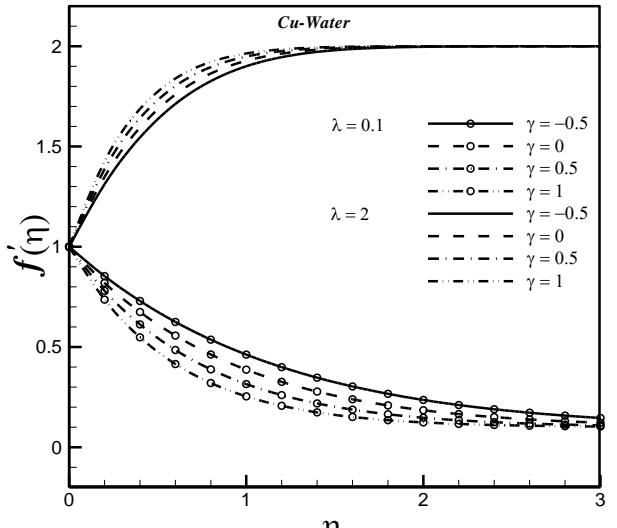

(a)

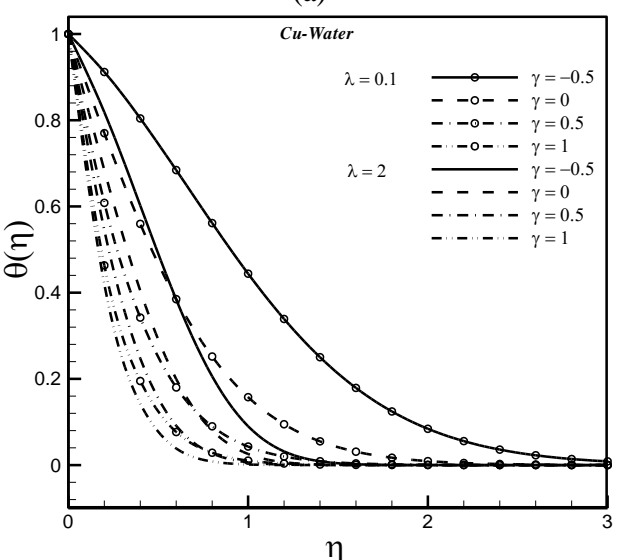

(b)

Fig. 3. Effect of Suction or injection parameter $(\gamma)$ and nanoparticle volume fraction $(\phi)$ on Velocity profile and Temperature distribution when $K_{1}=0.1, \phi=0.1, \varepsilon=0.1$ and $\operatorname{Pr}=6.2$.

Table 4 shows that the effects of Permeability parameter, Suction/injection parameter, velocity ratio parameter and nanoparticle volume fraction on skin friction coefficient. Physically, negative sign of $\tilde{C}_{f}$ implies that the stretching tube exerts a dragging force on the fluid and positive sign implies the opposite. For both suction $(\gamma>0)$ or injection $(\gamma<0)$ and both cases of $\lambda(\lambda=0.1$ or $\lambda=2)$ it can be seen that the absolute values of skin friction increases due to increase in permeability parameter and suction/injection parameter, while it decreases due to decrease in nanoparticle volume fraction.

Table 5 shows that the effects of permeability parameter, Suction/injection parameter, Velocity ratio parameter and nanoparticle volume fraction on Nusselt number. When $\lambda=0.1$ and $\lambda=2$, for suction, Nusselt number decreases with increasing in nanoparticle volume fraction, whereas the opposite trend is observed for injection. For suction or injection, Nusselt number increases due to increase the Permeability parameter when $\lambda=2$, but opposite behavior is noted when $\lambda=0.1$. However, increasing in $\gamma$ leads to increase in Nusselt number for both the cases of $\lambda$. 
Table 6 shows that the effects of Velocity ratio parameter and nanoparticle volume fraction on skin friction coefficient and Nusselt number. When $\lambda<1$, absolute values of skin friction decrease with increase of $\lambda$, while it increases when $\lambda>1$. Also, for both suction and injection and any values of $\lambda$ it can be found that Nusselt number increases as velocity ratio parameter increases. Table 7 shows variation Variation in Nusselt number with different base fluids. For fixed values of nanoparticle volume fraction, selecting Ethylene glycol instead of Water as base fluid lead to decrease thermal boundary layer thickness, so, $\mathrm{Cu}-$ Ethylene glycol has higher Nusselt number than $\mathrm{Cu}-$ Water due to lower thermal conductivity.

Table 5 Effects of Permeability parameter, Suction/injection parameter and nanoparticle volume fraction on Nusselt number

when $\varepsilon=0.1$ and $\operatorname{Pr}=6.2$ for $\mathrm{Cu}$-Water.

\begin{tabular}{|c|c|c|c|c|c|}
\hline \multirow{2}{*}{$K_{I}$} & \multirow{2}{*}{$\gamma$} & \multicolumn{2}{|c|}{$\lambda=0.1$} & \multicolumn{2}{|c|}{$\lambda=2$} \\
\cline { 3 - 6 } & & \multicolumn{4}{|c|}{$\phi$} \\
\cline { 3 - 6 } & & 0 & 0.05 & 0 & 0.05 \\
\hline 0.1 & -0.5 & 0.3364 & 0.3740 & 0.7406 & 0.7830 \\
\hline 0.1 & 0.5 & 3.7979 & 2.8177 & 4.1515 & 3.1838 \\
\hline 1 & 0.5 & 3.7658 & 2.7892 & 4.162 & 3.1921 \\
\hline 0.5 & -0.5 & 0.3052 & 0.3468 & 0.7471 & 0.787 \\
\hline 1 & -0.5 & 0.2715 & 0.3167 & 0.7546 & 0.7936 \\
\hline
\end{tabular}

Table 6 Effects of Velocity ratio parameter and nanoparticle volume fraction on skin friction coefficient and Nusselt number

when $\varepsilon=0.1, K_{1}=0.1$ and $\operatorname{Pr}=6.2$ for $\mathrm{Cu}$-Water.

\begin{tabular}{|c|c|c|c|c|c|}
\hline \multirow{2}{*}{$\gamma$} & \multirow{2}{*}{$\lambda$} & \multicolumn{2}{|c|}{$\tilde{C}_{f}$} & \multicolumn{2}{|c|}{$\tilde{N} u_{x}$} \\
\cline { 3 - 6 } & & \multicolumn{4}{|c|}{$\phi$} \\
\cline { 3 - 6 } & & 0 & 0.05 & 0 & 0.05 \\
\hline 0.5 & 0.1 & -1.263 & -1.256 & 3.7979 & 2.8177 \\
\hline 0.5 & 2 & 2.3337 & 2.3293 & 4.1515 & 3.1838 \\
\hline-0.5 & 0.1 & -0.805 & -0.797 & 0.3364 & 0.3740 \\
\hline-0.5 & 2 & 1.7784 & 1.773 & 0.7406 & 0.7830 \\
\hline
\end{tabular}

Table 7 Variation in Nusselt number with different base fluids (Water $\operatorname{Pr}=6.2$ and Ethylene glycol $P r=203.6$ ) when $K_{l}=0.1, \gamma=0.5, \lambda=0.1$.

\begin{tabular}{|c|c|c|c|c|}
\hline \multirow{2}{*}{$\varepsilon$} & \multicolumn{2}{|c|}{$C u$-Water } & \multicolumn{2}{c|}{$C u$-Ethylene glycol } \\
\cline { 2 - 5 } & \multicolumn{4}{|c|}{$\phi$} \\
\cline { 2 - 5 } & 0 & 0.05 & 0 & 0.05 \\
\hline 0 & 4.1383 & 3.0666 & 103.70 & 76.985 \\
\hline 0.1 & 3.7979 & 2.8177 & 94.35 & 70.069 \\
\hline 0.5 & 2.8837 & 2.1477 & 69.440 & 51.622 \\
\hline 1 & 2.2459 & 1.6784 & 52.307 & 38.936 \\
\hline
\end{tabular}

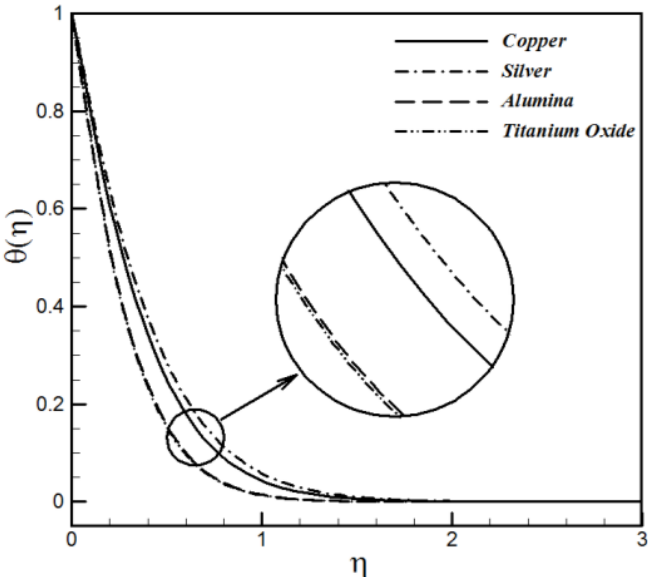

(a)

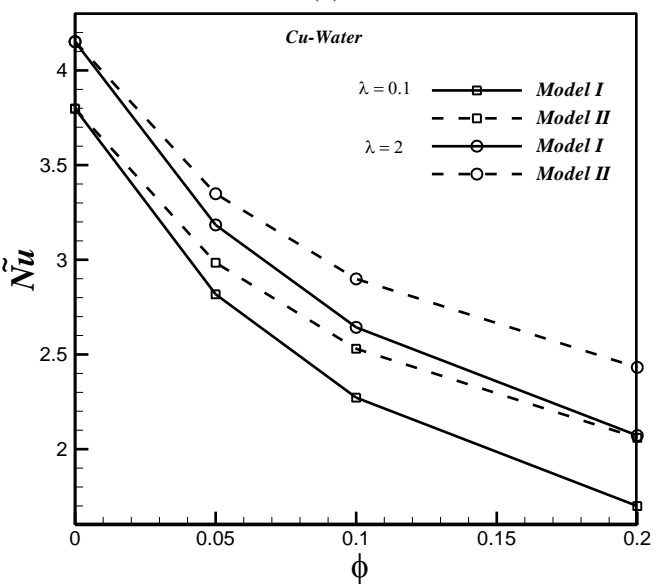

(b)

Fig. 4.(a) Temperature distribution for different types of nanofluids when $K_{I}=0.1, \phi=0.1, \varepsilon=0.1, \gamma=0.5$

,$\lambda=0.1$ and $\operatorname{Pr}=6.2$; (b) Variation in Nusselt number with nanoparticle volume fraction for different models $K_{1}=0.1, \varepsilon=0.1, \gamma=0.5$ and $\operatorname{Pr}=6.2$.

Tables 8 and 9 display the effect of different kinds of nanoparticle on skin friction coefficient and Nusselt number. Figure 4(a) shows that temperature distribution for different types of nanofluids. By using different types of nanofluid the value of $\tilde{C}_{f}$, $\tilde{N} u_{x}$ and temperature profile change. This means that the nanofluids will be important in the cooling and heating processes. Choosing Titanium oxide as the nanoparticle leads to the maximum amount rate of hate transfer, while selecting Silver leads to the minimum amount of it. Also, choosing Alumina as the nanoparticle leads to the maximum amount skin friction coefficient, while selecting Silver leads to the minimum amount of it. In Fig. 4(b), the Nusselt numbers versus the volume fraction of nanoparticle is shown when different models of nanofluid based on different formulas for thermal conductivity and dynamic viscosity is used. For all amount of $\lambda$, model $I I$ for nanotubes has higher Nusselt number than model $I$ for spherical shaped nanoparticles (due to lower thermal conductivity). 
Table 8 Effects of the nanoparticle volume fraction for different types of nanofluids on skin friction coefficient when $M=1$ and $\lambda=0.1$.

\begin{tabular}{|c|c|c|c|c|c|}
\hline & & \multicolumn{4}{|c|}{ nanoprticles } \\
\hline$\lambda$ & $\phi$ & $\mathrm{Cu}$ & $\mathrm{Ag}$ & $\mathrm{Al}_{2} \mathrm{O}_{3}$ & $\mathrm{TiO}_{2}$ \\
\hline 0.1 & 0.05 & -1.263 & -1.254 & -1.263 & -1.263 \\
\hline 0.1 & 0.1 & -1.253 & -1.250 & -1.263 & -1.263 \\
\hline 2 & 0.05 & 2.329 & 2.3283 & 2.3334 & 2.3331 \\
\hline 2 & 0.1 & 2.327 & 2.3258 & 2.3337 & 2.3332 \\
\hline
\end{tabular}

Table 9 Effects of the nanoparticle volume fraction for different types of nanofluids on Nusselt number

when $S=0.1, n=1, M=1, \lambda=0.1$ and $\operatorname{Pr}=6.2$.

\begin{tabular}{|c|c|c|c|c|c|}
\hline & & \multicolumn{4}{|c|}{ nanoprticles } \\
\hline$\lambda$ & $\phi$ & $\mathrm{Cu}$ & $\mathrm{Ag}$ & $\mathrm{Al}_{2} \mathrm{O}_{3}$ & $\mathrm{TiO}_{2}$ \\
\hline 0.1 & 0.05 & 3.797 & 2.668 & 3.312 & 3.329 \\
\hline 0.1 & 0.1 & 2.272 & 2.082 & 2.961 & 2.995 \\
\hline 2 & 0.05 & 3.183 & 3.036 & 3.673 & 3.689 \\
\hline 2 & 0.1 & 2.643 & 2.454 & 3.327 & 3.360 \\
\hline
\end{tabular}

\section{CONCLUSIONS}

In the present paper two-dimensional laminarforced convection nanofluids flow over a stretching surface at a porous surface has been numerically studied. The effects of the thermal conductivity parameter, volume fraction of the nanoparticles, permeability parameter, suction/injection parameter and velocity ratio parameter on the flow and heat transfer characteristics are determined for four kinds of nanofluids: copper, silver, titanium oxide and alumina. The working fluid is water and this base fluid is compared with Ethylene glycol. Two different models of nanofluid based on different formulas for thermal conductivity and dynamic viscosity are used. From this investigation, some conclusions were summarized as follows:

a. Fluid temperature increases due to increase in the thermal conductivity parameter, volume fraction of the nanoparticles while it decreases due to increase in the Velocity ratio parameter.

b. Absolute values of skin friction increases due to increase in suction/injection parameter, Permeability parameter and Velocity ratio parameter (when $\lambda>1$ ), while decreases due to increase in volume fraction of the nanoparticles and Velocity ratio parameter (when $\lambda<1$ ).

c. Nusselt number has direct relationship with Velocity ratio parameter, suction/injection parameter, Permeability parameter (when $\lambda>1$ ) and volume fraction of the nanoparticles (for injection) but it has reverse relationship with Permeability parameter (when $\lambda<1$ ) and volume fraction of the nanoparticles (for suction).

d. Choosing Titanium oxide as the nanoparticle proved to have the highest cooling performance for this problem. Also, selecting Ethylene glycol as base fluid lead to obtain higher Nusselt number.

e. The use of nanotubes (cylindrical shaped nanoparticles) in model II proved to have higher heat transfer enhancement as compared to spherical shaped nanoparticles (model $I$ ).

\section{REFERENCES}

Afzal, N., I.S. Varshney (1980). The cooling of a low resistance stretching sheet moving through a fluid, Heat Mass Transfer 14, 289-293.

Afzal, N. (1993). Heat transfer from a stretching surface, International Journal of Heat and Mass Transfer 36, 1128-1131.

Al-Sanea, S.A. (2004). Mixed convection heat transfer along a continuously moving heated vertical plate with suction or injection. International Journal of Heat and Mass Transfer 47, 1445-1465.

Choi, S (1995). Enhancing thermal conductivity of fluids with nanoparticle in: D.A.Siginer, H.P. Wang (Eds.), Developments and Applications of Non-Newtonian Flows. ASME MD 231, 99105.

Ellahi, R. (2013). The effects of MHD and temperature dependent viscosity on the flow of non-Newtonian nanofluid in a pipe: Analytical solutions. Applied Mathematical Modelling 37 (3), 1451-1467.

Grubka, L.J. and K.M. Bobba (1985). Heat transfercharacteristics of a continuously stretching surface with variable temperature. Transactions of ASME Journal of Heat and Mass Transfer 107, 248-250.

Ghasemi, E., Soheil Soleimani, H. Bararnia (2012). Natural convection between a circular enclosure and an elliptic cylinder using Control Volume based Finite Element Method, International Communications in Heat and Mass Transfer 39, 1035-1044.

Ghasemi, E., D.M. McEligot, K.P. Nolan, J. Crepeau, A. Tokuhiro, R.S. Budwig (2013). Entropy generation in a transitional boundary layer region under the influence of Free stream turbulence using transitional RANS models and DNS. International Communications in Heat and Mass Transfer 41, 10-16. 
Khan, W.A., A. Aziz (2011).Natural convection flow of a nanofluid over a vertical plate with uniform surface heat flux. International Journal of Thermal Sciences 50(7), 12071214.

Ramiar, A., A.A. Ranjbar and S.F. Hosseinizadeh (2012). Effect of Axial Conduction and Variable Properties on Two-Dimensional Conjugate Heat Transfer of $\mathrm{Al}_{2} \mathrm{O}_{3}$-EG/Water Mixture Nanofluid in Microchannel, Journal of Applied Fluid Mechanics (5) 3, 79-87.

Rashad, A. M., A. J. Chamkha and M. M. M. Abdou (2013). Mixed Convection Flow of Non-Newtonian Fluid from Vertical Surface Saturated in a Porous Medium Filled with a Nanofluid. Journal of Applied Fluid Mechanics (6) 2, 301-309.

Sharma, P.R. and G. Singh (2009). Effects of Variable Thermal Conductivity and Heat Source / Sink on MHD Flow Near a Stagnation Point on a Linearly Stretching Sheet. Journal of Applied Fluid Mechanics (2)1, 13-21.

Soleimani, S., M. Sheikholeslami, D.D. Ganji and M. Gorji-Bandpay (2012). Natural convection heat transfer in a nanofluid filled semi-annulus enclosure. International Communications in Heat and Mass Transfer 39, 565-574.

Sheikholeslami, M., M. Gorji-Bandpay, D.D. Ganji (2012). Magnetic field effects on natural convection around a horizontal circular cylinder inside a square enclosure filled with nanofluid. International Communications in Heat and Mass Transfer 39, 978-986.

Sheikholeslami, M., M. Gorji-Bandpy, D.D. Ganji, Soheil Soleimani, S.M. Seyyedi (2012). Natural convection of nanofluids in an enclosure between a circular and a sinusoidal cylinder in the presence of magnetic field. International Communications in Heat and Mass Transfer 39, 1435-1443.

Sheikholeslami, M., M. Gorji-Bandpy, G. Domairry (2013). Free convection of nanofluid filled enclosure using lattice Boltzmann method (LBM), Applied Mathematics and Mechanics English edition 34(7), 1-15.

Sheikholeslami, M., M. Gorji-Bandpy, S.M. Seyyedi, D.D. Ganji, Houman B. Rokni and Soheil Soleimani (2013). Application of LBM in simulation of natural convection in a nanofluid filled square cavity with curve boundaries. Powder Technology 247, 87-94.

Sheikholeslami, M., M. Gorji-Bandpy, D.D. Ganji (2013). Natural convection in a nanofluid filled concentric annulus between an outer square cylinder and an inner elliptic cylinder. Scientia Iranica, Transaction B: Mechanical Engineerin 20(4), 1-13.

Sheikholeslami, M., M. Gorji-Bandpy, Soheil Soleimani (2013). Two phase simulation of nanofluid flow and heat transfer using heatline analysis. International Communications in Heat and Mass Transfer doi.org/10.1016/j.icheatmasstransfer.2013.07.0 06.

Sheikholeslami, M., M. Gorji-Bandpy, D. D. Ganji (2013). Numerical investigation of MHD effects on Al2O3-water nanofluid flow and heat transfer in a semi-annulus enclosure using LBM. Energy, DOI: 10.1016/j.energy.2013.07.070.

Sheikholeslami, M. and D.D. Ganji (2013). Heat transfer of $\mathrm{Cu}$-water nanofluid flow between parallel plates, Powder Technology 235, 873879.

Sheikholeslami, M., M. Gorji-Bandpy, D.D. Ganji , Soheil Soleimani (2013). Natural convection heat transfer in a cavity with sinusoidal wall filled with $\mathrm{CuO}$-water nanofluid in presence of magnetic field. Journal of the Taiwan Institute of Chemical Engineers, doi.org/10.1016/j.jtice.2013.04.019.

Sheikholeslami, M., M. Gorji-Bandpy, D.D. Ganji, Soheil Soleimani (2013). Effect of a magnetic field on natural convection in an inclined halfannulus enclosure filled with $\mathrm{Cu}$-water nanofluid using CVFEM. Advanced Powder Technology doi.org/10.1016/j.apt.2013.01.012.

Vajravelu, K., K.V. Prasad , J. Lee, Ch. Lee, I. Pop, R. A. Van Gorder (2011). Convective heat transfer in the flow of viscous Agewater and $\mathrm{Cu}$-water nanofluids over a stretching surface. International Journal of Thermal Sciences 50, 843-851.

Yacob, N.A., A. Ishak, I. Pop (2011). FalknereSkan problem for a static or moving wedge in nanofluids. International Journal of Thermal Sciences 50, 133-139. 